\title{
Self-assembly processes of superparamagnetic colloids in a quasi-two-dimensional system
}

\author{
N. Rojas, ${ }^{*}$ A. Darras, ${ }^{\dagger}$ and G. Lumay \\ GRASP-CESAM, University of Liège, B-4000 Liège, Belgium
}

(Received 29 April 2017; published 24 July 2017)

\begin{abstract}
Superparamagnetic colloids gather depending on the magnitude of the magnetic field applied, forming chains and ribbons in a quasi-two-dimensional chamber. The results presented in this work are in good agreement with recent experimental multistable data for the mean length of the aggregates in thermodynamic equilibrium.
\end{abstract}

DOI: 10.1103/PhysRevE.96.012608

\section{INTRODUCTION}

Magnetic colloids have been extensively studied in the framework of ferrofluids. In pioneer works [1,2], the equation of state and the static correlations of ferromagnetic grains were studied. It was shown that monomers aggregate forming chains if the density of ferromagnetic particles is enough low and if the dipole-dipole coupling is sufficiently strong. In Ref. [3] a theory for a strongly dipolar fluid at low densities is developed; the free energy as a functional of the chain length distribution provides expressions for the energy and conformal entropy of a long dipole chain considering very weak interchain interactions. In Ref. [4] extensive Monte Carlo (MC) simulations of a dipolar hard-sphere fluid are performed in order to investigate the chain structure at low densities. MC simulations reveal that self-assembled chains break up and recombine in typical equilibrium runs. In Ref. [5] the dipolar forces that affect the structure and phase behavior of the colloidal suspension are studied. In the present work, some of the results for the mean length of the chains are similar to the approximations obtained in the latter reference [see Eq. (16) therein]. In Ref. [6] by considering straight and rigid chains the effect of chain-shaped aggregates is studied, in which the rheological properties of ferrocolloids and the characteristic time of hydrodynamic relaxation are reviewed. In Ref. [7] an investigation of spatial and orientational intrachain correlations and flexibility allows the extension of basic concepts in polymer physics. Some of the analytic results for the mean length of the chains obtained in the present article are similar to the results in the latter reference [see Eqs. (8) and (9) therein]. In Ref. [8] it is shown that the aggregation occurs for large enough number of particles. In Ref. [9] the chain distribution is obtained with a free energy minimization method, neglecting interchain interactions. A higher ferrofluid magnetization (in comparison to the Langevin magnetization) due to chain orientational correlations and chain lengthening is obtained. The equilibrium length lengthening is solved analytically in the case of noninteracting flexible chains and low concentration magnetic fluid. The flexible chain orientational response is shown to be weaker than in rigid rodlike chains. In Ref. [10] the model takes into account a polydisperse suspension; the formation of soft heterogeneous

\footnotetext{
*Present address: Service de Physique de l'Etat Condensé, CEA Saclay, France; nicolas.rojas@email.com.

${ }^{\dagger}$ F.R.S.-FNRS, B-1000 Bruxelles, Belgium; Experimental Physics, Saarland University, D-66123 Saarbrücken, Germany.
}

chains occurs if sufficiently big particles are present. The suspension is composed of large ferromagnetic particles and small superparamagnetic grains, taking into account that the effective liquid has no magnetoviscous properties. In Ref. [11] the scenarios of condensation phase transition in ferrofluids are studied theoretically, and the results show that the appearance of linear chains precedes the magnetic particle bulk condensation.

In Refs. $[12,13]$ the aggregation process of superparamagnetic colloids under an external magnetic field has been studied, with an interesting finding: in thermodynamic equilibrium the formation of chains leads to stable structures such as ribbons when the magnetic field is increased. Superparamagnetic colloids under a constant magnetic field $\mathbf{B}=B \hat{y}$ in a two-dimensional system building arrays of particles oriented in the direction of the magnetic field have been found experimentally and in numerical simulations $[14,15]$. The dynamical transient that leads to saturated aggregates in thermodynamic equilibrium has been extensively studied. In Refs. $[16,17]$ the Smoluchowsky equation is solved using a numerical method described in Ref. [18], employing a kernel for magnetic-field-induced aggregation $k_{i j}^{\text {mag }}$ that depends on the diameter of the particles, the critical angle $\theta_{m}=54.7^{\circ}$, the average translational diffusion coefficient of rigid rods, the kinetic viscosity of the liquid phase, and the thermal energy $k_{B} T$. The latter considers a mean field approach that takes into account long-range and anisotropic dipole-dipole interactions. The theory is in good agreement with experimental measurements of $X_{n}$ (the concentration of clusters containing $n$ primary particles $\phi_{n}$, normalized by the initial monomer concentration $\left.\phi_{0}\right)$, and the weight-average chain length of the aggregates $\bar{s}(t)$, as a function of the exposure time to the magnetic field. It is noticed that the latter approach depends on a free parameter $\bar{h}$ (the effective interaction range averaged over the attractive region), and the long time saturated regime is not addressed. Later, the transient and long range equilibrium dynamics are obtained with Langevin dynamics simulations $[14,19,20]$ and in experimental results $[12,13,16,17,21]$. The evolution of the mean length of the aggregates $\bar{s}(t)$ scales with $t^{z}$ [15] until the saturation regime, where it converges to a constant value $[\bar{s}(t) \rightarrow \bar{s}]$ in thermodynamic equilibrium after a transient time $\left(t \rightarrow t_{\mathrm{sat}}\right)$. The exponent $z$ and the saturation value $\bar{s}$ depend on the magnitude of the magnetic field. In the latter regime, the aggregates interact and exchange particles as a typical behavior of the dynamic equilibrium, while the mean length $\bar{s}$ in the system remains roughly constant; these are called saturated aggregates. 
Recently [12], in addition to chains, ribbons are found when the magnetic field magnitude is increased in a quasitwo-dimensional chamber. In this regime the experiment is very sensitive to external noise. For instance, the flow around a small moving air or oil bubble accidentally trapped in the colloidal suspension can influence the growth process of the aggregates. Then each experimental realization leads to a different value of $\bar{s}$ at constant $B$ in the range $B^{2}=200$ to $700 \mathrm{G}^{2}$, which provides a multistable region for the mean length of the aggregates. The system is quasi-two-dimensional, the diameter of the cylindrical chamber is $5 \mathrm{~mm}$, with a height of approximately $50 \mu \mathrm{m}$, the radius of the particles is $0.6 \pm 0.3 \mu \mathrm{m}$, then the aspect ratio of the system is about $10^{-2}$. The present work will be focused on describing recent experimental findings [12], in the range of parameters of stable ribbons in thermodynamic equilibrium. The most important results in this paper are summarized in Sec. III E where multistable experimental data for the mean length of superparamagnetic ribbons is described by the multiplicity of the numerical results, which increase with the magnetic field magnitude.

\section{REVIEW}

In this section a review of the theory that explains saturated chains in thermodynamic equilibrium will be presented. As a starting point the Gibbs free energy [22] of the system is written

$$
F=\sum_{s^{\prime}=1}^{\infty}\left[-\frac{N_{s^{\prime}}}{s^{\prime}}\left(s^{\prime}-1\right) \epsilon_{m}+k_{B} T \frac{N_{s^{\prime}}}{s^{\prime}} \log \left(\frac{N_{s^{\prime}}}{s^{\prime} A}\right)\right]+F_{0},
$$

where the first term in the sum is the energy of the chains of length $s^{\prime}$, the second term is $-T \times$ the entropy of chains of length $s^{\prime}$, and $F_{0}$ is any other contribution to the free energy. The length $s^{\prime}$ is measured in number of grains $s^{\prime}=1,2, \ldots$. Here $-\epsilon_{m}$ is the magnetic energy of each first order (nearest neighbor) bond in a chain, $N_{s^{\prime}}$ is the number of grains in chains of length $s^{\prime}, A=\sum_{s^{\prime}=1}^{\infty} A_{s^{\prime}}$ is the total number of chains in the suspension, where $A_{s^{\prime}}$ is the number of chains of length $s^{\prime}, k_{B}$ is the Boltzmann constant, and $T$ is the temperature of the system. To compute the chemical potential of an aggregate $\mu_{s}=\left.\left(\partial F / \partial N_{s}\right)\right|_{\left(N_{s} \neq N_{s^{\prime}}, T, V\right)}$ employing (1), the use of Kronecker delta conventions is required, which yields after calculation

$$
\mu_{s}=-\frac{(s-1)}{s} \epsilon_{m}+\frac{k_{B} T}{s} \log \left(\frac{N_{s}}{s A}\right)+\mu_{0}
$$

with $\mu_{0}=\left.\left(\partial F_{0} / \partial N_{s}\right)\right|_{\left(N_{s} \neq N_{s^{\prime}}, T, V\right)}$, and $V$ is the volume of the suspension. The volume fraction in absence of magnetic field (before aggregation) $\phi_{0}=N V_{\odot} / V$ is equal to the volume fraction of particles in presence of magnetic field (after aggregation), which is equivalent to the conservation of grains in the system

$$
\phi_{0}=\sum_{s=1}^{\infty} \phi_{s}
$$

where $V_{\odot}$ is the volume of each colloidal particle, $N$ is the total number of grains (which is a conserved quantity), and $\phi_{s}=N_{s} V_{\odot} / V$ is the volume fraction of particles in chains of length $s$. In the case of ribbons, their width is given by the parameter $d$, measured in number of grains $(d=2,3,4)$. Volume fractions $\phi_{0}$ and $\phi_{s}$ are smaller than 1 since the total volume of colloidal particles is smaller than the volume of the suspension. Equation (2) can be rewritten using $\mu_{s}^{0}$, the standard part of the chemical potential (the mean interaction free energy per particle)

$$
\mu_{s}=\frac{1}{s}\left[k_{B} T \log \left(\frac{\phi_{s}}{s}\right)-(s-1) \epsilon_{m}\right]+\mu_{s}^{0}
$$

with $\mu_{s}^{0}=\mu_{0}+\alpha k_{B} T / s$ and $\alpha=\log \left(V / A V_{\odot}\right)$. In the case of chains, $V / A V_{\odot}=V \bar{s} / V_{p}>1$ using $\bar{s}=N / A$ and $V_{p}=$ $N V_{\odot}$ the total volume occupied by the grains, then $\alpha>0$. In the present work $\bar{s}$ is the saturated mean length of the aggregates in thermodynamic equilibrium; for the dynamics of growing aggregates the notation $\bar{s}(t)$ will be used. To understand the use of $\mu_{s}^{0} \equiv \mu^{0}$ as a constant in Ref. [14], a review of Ref. [22] (chapter 16) is important. In thermodynamic equilibrium the chemical potential of all the aggregates is equal, stated by the equation $\mu_{s}=\mu_{1}$. The rate of association of aggregates is given by $k_{1} \phi_{1}^{s}$, which neglects, for instance, the possible formation of a pentamer by the union of a dimer with a trimer. The rate of dissociation is $k_{s} \phi_{s} / s$, this implies for example, that $s$-mers can only completely dissociate to form monomers $(s=1)$, but not a $(s-1)$-mer and a monomer. Also, a pentamer is not allowed to break to form a dimer and a trimer. The ratio of the reaction rates is defined by $K_{s}=k_{1} / k_{s}$, known as the equilibrium constant according to the generalized mass action law. The calculation is simplified by considering that the particles have no magnetic interaction $\left(\epsilon_{m}=0\right)$. In thermodynamic equilibrium, equations $\mu_{1}=\mu_{s}$ and (4) state

$$
\phi_{s}=s\left(\phi_{1} e^{\beta\left(\mu_{1}^{0}-\mu_{s}^{0}\right)}\right)^{s}=s \phi_{1}^{s} K_{s},
$$

where $\beta=1 / k_{B} T$. The last equality in (5) implies that the rate of association is equal to the rate of dissociation; this is known as ideal mixing and is restricted to dilute systems. Then, for instance, the model cannot capture an equilibrium state in the absence of monomers, where larger aggregates may coexist in equilibrium. Considering $\mu_{s}^{0}$ as a constant is equivalent to impose $K_{s}=1$, which is an approximation that favours monomers since it yields $0<\phi_{s} \ll \phi_{1}<1$. If $\mu_{s}^{0}$ (in)decreases with $s$, Eq. (5) shows that the occurrence of large chains becomes (less)more probable. In the chains case $\mu_{s}^{0}=\mu_{0}+\alpha k_{B} T / s$ decreases with $s$ and the occurrence of large aggregates becomes more probable. The equilibrium constant is $K_{s}=e^{\alpha(s-1)} \geqslant 1$ since $\alpha>0$ and $s \geqslant 1$, regardless of the value of $\mu_{0}$. In Ref. [14] the aggregation process is considered mainly magnetic $\left(K_{s}=1, \epsilon_{m}>0\right)$, following a similar calculation as in (1)-(5), a useful variable $x=\phi_{1} e^{\beta \epsilon_{m}}$ allows us to write (3) as

$$
\phi_{0} e^{\beta \epsilon_{m}}=\sum_{s=1}^{\infty} s x^{s}=\frac{x}{(1-x)^{2}}
$$

with $0<x<1$. In the latter reference, the interaction energy $\epsilon_{m}$, has been shown to be proportional to $\Gamma-1=\beta \epsilon_{m}$ by averaging the magnetic interaction energy

$$
U(r, \theta)=\frac{4 \Gamma R^{3}}{\beta}\left(\frac{1-3 \cos ^{2} \theta}{r^{3}}\right)
$$


over the attractive region in spherical coordinates, where $\Gamma=\pi \chi^{2} R^{3} B^{2} / 9 \bar{\mu}_{0} k_{B} T$ is the ratio between the magnetic energy and the thermal energy, and $\Gamma / \beta$ is the magnitude of the magnetic potential evaluated in $r=2 R$ and $\theta=0$ (two particles in contact in a chain). The potential (7) has azimuthal symmetry. Here $\bar{\mu}_{0}$ is the vacuum permeability; this notation is used in order to avoid confusion with the chemical potential $\mu_{0}, \chi$ is the magnetic susceptibility and $R$ is the mean radius of the grains in the suspension. It is noticed that in Ref. [12] the approximation $\beta \epsilon_{m} \approx \Gamma$ is employed, i.e., without considering averages on the magnetic potential, which also will be used in the present work. In the case $\phi_{0} e^{\beta \epsilon_{m}} \ll 1$, the decrease of the interaction energy $\left(\epsilon_{m} \rightarrow 0\right)$ in Eq. (6) yields $\phi_{0}=x$ since there is no formation of chains, in this case $\phi_{0}=\phi_{1}$. In the case $\phi_{0} e^{\beta \epsilon_{m}} \gg 1(x \rightarrow 1)$, Eq. (6) gives

$$
x \simeq 1-\frac{1}{\sqrt{\phi_{0} e^{\beta \epsilon_{m}}}} .
$$

The number of chains of length $s, A_{s}=N_{s} / s=\phi_{s} V / s V_{\odot}$ can be expressed as $A_{s}=\phi_{1} x^{s} V / x V_{\odot}[14]$.

\section{SATURATED AGGREGATES}

\section{A. Chains}

The saturated mean length of the chains at equilibrium is computed as an average over all the chains

$$
\bar{s}=\frac{1}{A} \sum_{s=1}^{\infty} s A_{s}=\frac{\sum_{s=1}^{\infty} s x^{s}}{\sum_{s=1}^{\infty} x^{s}} .
$$

By inspection, Eq. (9) can be written as

$$
\bar{s}=\frac{x \sum_{s=1}^{\infty} s x^{s-1}}{\sum_{s=1}^{\infty} x^{s}}=x \frac{d}{d x} \log \left(\frac{x}{x-1}\right),
$$

and then $\bar{s}=(1-x)^{-1}=\sqrt{\phi_{0} e^{\beta \epsilon_{m}}}$ by using (8). This result has been celebrated in Ref. [14] and is useful to describe the growth of the mean size of chains for low and moderate magnetic field magnitudes (see Fig. 1 and Fig. 2, black thick line). A superior limit is noticed due to $\bar{s}=(1-x)^{-1}<$ $x /(1-x)^{2}$ when $1 / 2<x<1$ (see Fig. 2). Then Eq. (8) provides a upper bound (upper dotted line in Figs. 1 and 2)

$$
\bar{s}<\phi_{0} e^{\beta \epsilon_{m}}\left(1-\frac{1}{\sqrt{\phi_{0} e^{\beta \epsilon_{m}}}}\right)<\phi_{0} e^{\beta \epsilon_{m}} .
$$

In the limit $x \rightarrow 1$ Eq. (11) is $\bar{s}<\bar{s}^{2}$, which is related to the minimum size of the chains, which cannot be lower than $\bar{s}=1$. The first inequality in (11) also can be written as $\bar{s}<\bar{s}^{2} x$, which gives a lower bound (lower dotted line in Figs. 1 and 2)

$$
1<\left(1-\frac{1}{\sqrt{\phi_{0} e^{\beta \epsilon_{m}}}}\right)^{-1}<\bar{s} .
$$

In Fig. 1 the expression $\phi_{0} e^{\beta \epsilon_{m}}$ is replaced by $C_{2}^{2} e^{C_{1} B^{2}}$ following previous findings on the average interaction energy $\epsilon_{m}$ and the volume fraction $\phi_{0}[12,13]$. The numerical values $C_{1}=4.4 \times 10^{-2} \mathrm{G}^{-2}$ and $C_{2}=2.1$ are employed. One of the problems of this approach is that the volume fraction before aggregation is $\phi_{0}=C_{2}^{2}>1$. Note that the bounds in Eqs. (11) and (12) are valid when $1 / 2<x<1$. At $x=0.5, \bar{s}$ and its

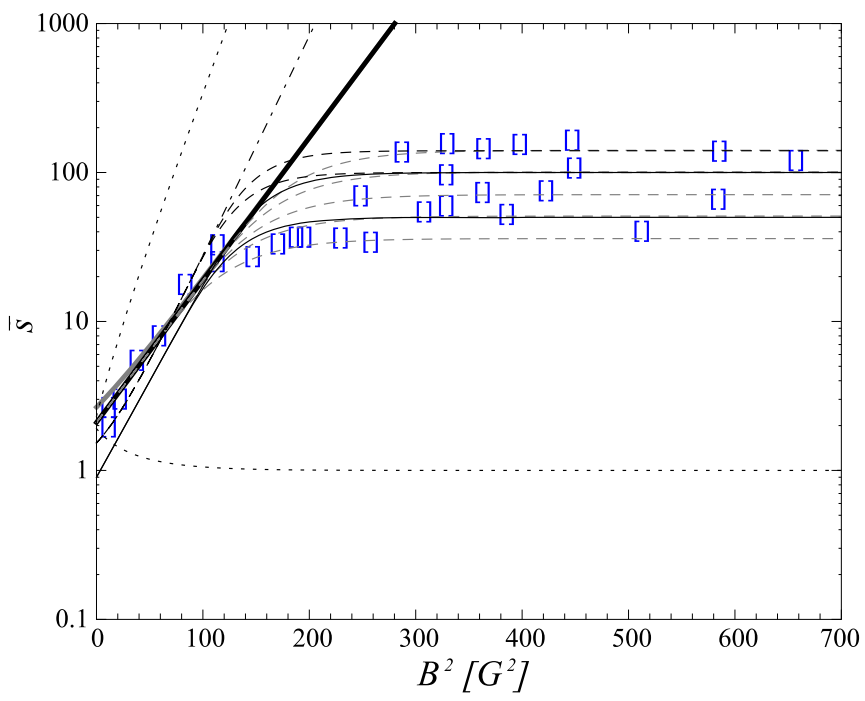

FIG. 1. Mean length of saturated chains and ribbons at thermodynamic equilibrium. Experimental data obtained from Refs. [12,13] [(blue) []]. Solution of Eq. (10) $\bar{s}=\sqrt{\phi_{0} e^{\beta \epsilon_{m}}}$ (black thick line) [14]. Upper limit provided by central formula in inequality (11) (upper dotted line). Lower bound given by Eq. (12) (lower dotted curve). Equation (13) for $\bar{s}_{\infty}=35,50,70,100,140$ (gray dashed curves). Complete first order solution (27) (gray thick curve) and previous approximations for $\phi_{0}=4.41$. Solutions $\bar{s}_{a 1}$ (33) (dot-dashed line), (34) for $\bar{s}_{\infty}=100,140$ (black dashed curves), and (35) for $\bar{s}_{\infty}=50,100$ (black thin curves) evaluated at $\phi_{0}=0.81$.

upper and lower limits converge to $\bar{s}=2$ as shown in Fig. 2 . Although the findings in [12] (see Fig. 4 therein and Fig. 1) are in agreement with experimental measurements [(blue) []] until $B^{2} \simeq 100 \mathrm{G}^{2}$, there is no theory for the data obtained above the latter value. One of the main quests on the experimental results is then related to describing the multistable region of mean lengths when the magnetic field magnitude is increased.

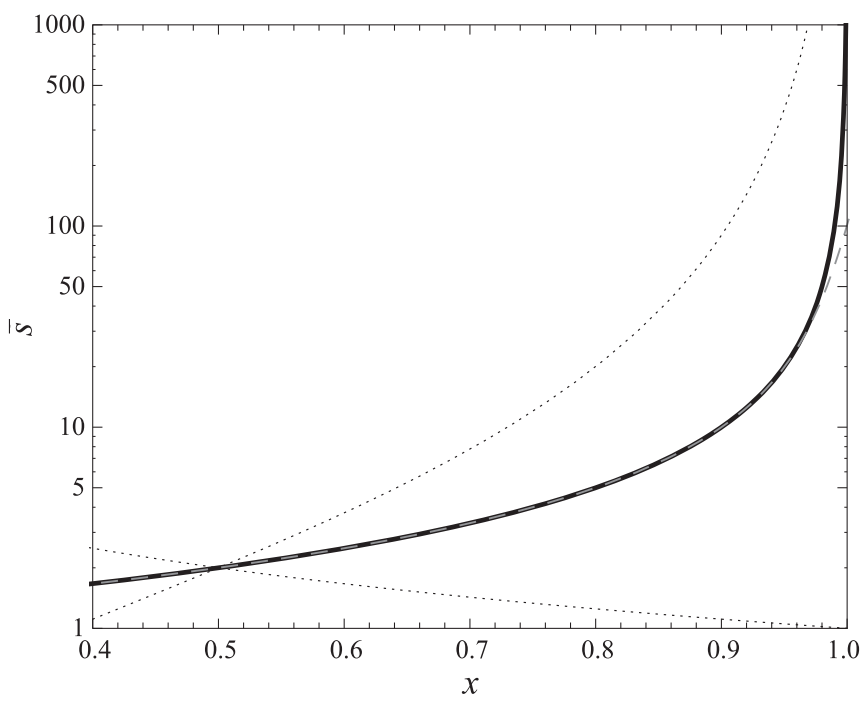

FIG. 2. Upper limit provided by central formula in inequality (11) (upper dotted line). Lower bound given by Eq. (12) (lower dotted curve). Solution of Eq. (10) (black thick line) using (8) [14]. Equation (13) for $\bar{s}_{\infty}=100$ (gray dashed curve). 
The result $\bar{s}=\sqrt{\phi_{0} e^{\Gamma}}$ is valid only for chains since it diverges when $B$ increases (black thick line in Figs. 1 and 2). Here it is shown a mathematical solution of the problem. $\bar{s}=(1-x)^{-1}$ is a good approximation when $x<1$, but when $x \rightarrow 1, \bar{s}$ diverges. A straightforward solution is given by using (8) and the series of $(1-x)^{-1}$ :

$$
\bar{s}=\frac{1}{1-x} \simeq \sum_{j=0}^{\bar{s}_{\infty}-1} x^{j} \simeq \sum_{j=0}^{\bar{s}_{\infty}-1}\left(1-\frac{1}{\sqrt{\phi_{0} e^{\beta \epsilon_{m}}}}\right)^{j} .
$$

Figures 1 and 2 (gray dashed curves) show Eq. (13) for different values of $\bar{s}_{\infty}$, the convergence value of $\bar{s}$ when $x \rightarrow 1(B \gg 1)$. Note that the series is truncated at $\bar{s}_{\infty}-1$. An estimation of $\bar{s}$ when $B$ increases is given by

$$
\lim _{x \rightarrow 1} \bar{s}=\sum_{j=0}^{\bar{s}_{\infty}-1} 1^{j}=\bar{s}_{\infty},
$$

which explains the equivalence between the order of the series and the convergence value $\bar{s} \rightarrow \bar{s}_{\infty}$. In this limit, using the exact result $\bar{s}=\sqrt{\phi_{0} e^{\beta \epsilon_{m}}}$ is equivalent to consider ribbons of infinite length, which is physically impossible since $N$ is a conserved quantity. The longest length of a chain in the system is $s=N$, in the thought case that all the particles are aggregated in one chain. In this case the mean length is equal to the length of the chain $\bar{s}=N$ [replacing $A_{s}=\delta_{s, N}$ in (9)]. The latter is not plausible in real experiments. The asymptotic mean length in thermodynamic equilibrium $\bar{s}_{\infty}$, can be given then as an experimental parameter by counting $A$, the total amount of chains and ribbons in the suspension, and computing $\bar{s}_{\infty}=N / A$. The agreement of the result (13) with experimental measurements in Fig. 1 is given in this case without any dependency on the width of the ribbons $d$. It will be shown in the following Sec. III B that a similar result can be obtained in the case of ribbons. First and second order magnetic interactions that provide stable aggregates will be considered in the following section. The asymptotic value $\bar{s}_{\infty}$ is criticized under the point of view that the magnetic field can not be increased up to $\infty$. The latter supports the suggestion that the multistable region could grow by increasing $B^{2}>700 \mathrm{G}^{2}$. It is remarked that the result given by Eqs. (13) and (14) is only a mathematical approach to the problem but does not constitute its solution, since it does not consider any aspect related to the physics of ribbons formation or the aggregates interaction in thermodynamic equilibrium, which will be shown in the following sections.

\section{B. Ribbons}

In the previous part an approach that describe the experimental outcomes is given by approximation of the series in the parameter $x$. Although the theoretical results fit the obtained data, the latter model is built for superparamagnetic chains in thermodynamic equilibrium, and it is not valid in order to describe the physics of ribbons in this regime. One of the main problems with the latter approximation in Sec. III A is that $\bar{s}_{\infty}$ is a fixed value considered as a free parameter given by experimental measures, then each gray dashed curve in Fig. 1 is given by a different value of $\bar{s}_{\infty}$. Calculating $\bar{s}_{\infty}$ for high magnitudes of the magnetic field in one experimental
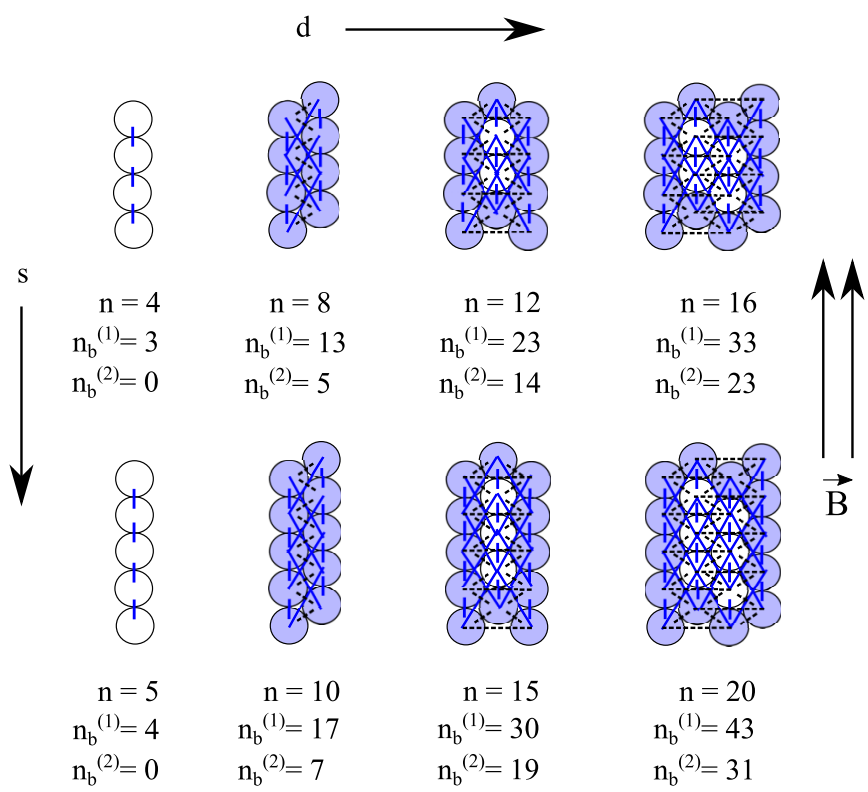

FIG. 3. Symmetric aggregates of length $s=4,5$ and width $d=1,2,3,4$ oriented in the direction of the magnetic field. The number of first order bonds (short lines) is given by $n_{b}^{(1)}=3(n-1)-$ $p=d(s-1)+(d-1)(2 s-1)$; the amount of second order bonds (long lines) is $n_{b}^{(2)}=(d-1)(2 s-3)+(d-2) s$. Perimeter grains $p$ are filled (blue color). Negative potential interactions are presented with solid (blue) lines and positive potential interactions with dashed lines.

realization will provide a curve within the ribbons region. This implies that the mean length of the ribbons $\bar{s}$ is a defined curve instead of a multistable region of mean lengths, as shown by the experimental results. In this section a similar to Eq. (9) will be computed with a dependency on $d$, the width of the ribbons. The equilibrium mean length $\bar{s}$ is now computed by counting $A$, the total amount of chains and ribbons in the suspension

$$
A=\sum_{d=1}^{d_{\max }} \sum_{s=d}^{\infty} A_{d, s}
$$

where $A_{d, s}$ is the number of chains or ribbons of length $s$ and width $d$, and $d_{\max }$ is the maximum width of a ribbon considered in the colloidal suspension; experimental observations state $d_{\max }=4$. Here the summation limits imply $A_{d, s}=0$ for $s<$ $d$, i.e., the aggregates considered in the computation of $\bar{s}$ are those oriented parallel to the magnetic field direction (see Figs. 3 and 4). The total number of grains in the suspension is given by

$$
N=\sum_{d=1}^{d_{\max }} \sum_{s=d}^{\infty} d s A_{d, s},
$$

which contains the implicit consideration that each aggregate has a number of particles $n=d s$ (symmetric approximation; see following text). The equation $\bar{s}=N / A$ is not valid in the case of ribbons. Equations (15) and (16) are employed for computing the mean number of particles of the aggregates $\bar{n}=N / A$ for any value in the range $0<x<1$. In order to compute the mean length of chains or ribbons, $s$ is averaged. For a sake of simplicity only symmetric aggregates are taken 

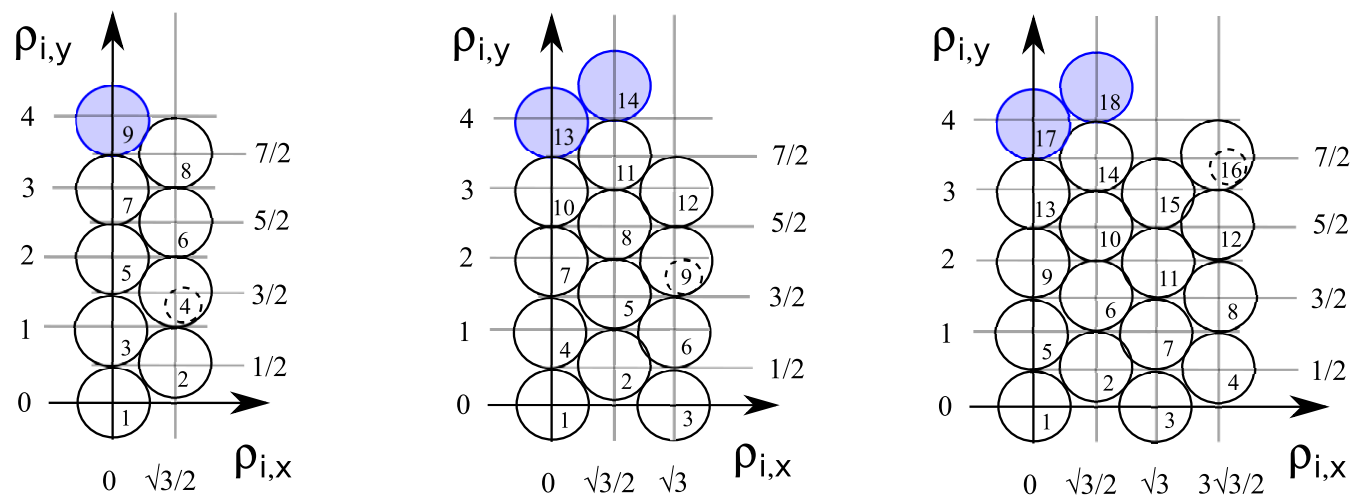

FIG. 4. Asymmetric ribbons labeled in nondimensional coordinates $\left(\rho_{i, x}, \rho_{i, y}\right)$ for $d=2,3,4$ and $s=4$. The rest $n$ mod $d$ particles are filled (blue). The particle $n_{0}=d^{2}$ (dashed line circles) indicates the smallest ribbon aligned parallel to the direction of the magnetic field, considered in the fit of the numerical results (29) employing $u_{d, n}=2\left(a_{d}-b_{d} n\right) / n$.

into account in this section, which means that each column in a ribbon has the same number of particles (or length) (see Fig. 3). Then the formula for $\bar{s}$ is

$$
\bar{s}=\frac{1}{A} \sum_{d=1}^{d_{\max }} \sum_{s=d}^{\infty} s A_{d, s} .
$$

The following part will be devoted to define $A_{d, s}$ as a function of $d, s$, and $x$. The number of particles in all the ribbons of length $s$ and width $d$ is $N_{d, s}=d s A_{d, s}$, the volume fraction $\phi_{d, s}=V_{\odot} N_{d, s} / V$ gives then $A_{d, s}=\phi_{d, s} V /\left(d s V_{\odot}\right)$. The volume fraction $\phi_{d, s}$ will be computed as a function of $\phi_{1,1}$ and $x=\phi_{1,1} e^{\beta \epsilon_{m}}$ by employing $\mu_{1,1}=\mu_{d, s}$ in thermodynamic equilibrium. The Gibbs free energy is

$$
F=F_{0}+\sum_{d^{\prime}=1}^{d_{\max }^{\prime}} \sum_{s^{\prime}=d^{\prime}}^{\infty} A_{d^{\prime}, s^{\prime}}\left[U_{d^{\prime}, s^{\prime}}-T S_{d^{\prime}, s^{\prime}}\right]
$$

where $U_{d^{\prime}, s^{\prime}}$ is the magnetic energy of the ribbons, $S_{d^{\prime}, s^{\prime}}$ is the entropy of the aggregates, and $F_{0}$ is any other contribution to the free energy. The magnetic energy is specified by counting $n_{b}^{(1)}$, the amount of bonds in a ribbon considering only nearest neighbors and $n_{b}^{(2)}$, the number of bonds at second order (see Fig. 3), third and higher order interactions are neglected in this calculation. Experimental observations state that one or two particles can sometimes aggregate on top of another agglomerate. Nevertheless, larger structures formed by two planes one on top of the other have not been observed; then the aggregates are mainly two-dimensional and rarely three-dimensional. Each chain and ribbon aggregate in a two dimensional plane; then the first order (short lines) and second order (long lines) distances are measured in the middle plane of the particles parallel to the direction of the magnetic field. In the chains case $\left(d_{\max }=1\right), U_{s}=-(s-1) \epsilon_{m}$ will be retrieved; in this case there are no positive interaction potentials. For chains and ribbons, first order interaction energies are negative only in the direction of the magnetic field. First, second and higher order neighbors balance the summation of attractive and repulsive forces in the aggregates. This is due to the form of the magnetic potential energy (7), which is negative for $\theta<\theta_{m}=54.7^{\circ}$ and positive for $\theta>\theta_{m}$. Equation (7) defines negative (solid (blue) lines) and positive (dashed lines) bonds, considering that the dipolar angle $\theta$ increases from the $y$ axis (magnetic field direction). The first order magnetic energies are

$$
\begin{gathered}
\epsilon_{m-}^{(1)}=\left.U\right|_{(r=2 R, \theta=0)}=-\frac{\Gamma}{\beta}, \\
\epsilon_{m+}^{(1)}=\left.U\right|_{(r=2 R, \theta=\pi / 3)}=\frac{\Gamma}{8 \beta} .
\end{gathered}
$$

In Refs. [12,13] and in this work the approximation $\epsilon_{m} \simeq \Gamma / \beta$ is employed, which is equivalent to ignore averages on the magnetic potential, defining $\epsilon_{m}$ as the magnitude of the magnetic energy for two particles in contact in a chain. The second order potentials are

$$
\begin{aligned}
& \epsilon_{m-}^{(2)}=\left.U\right|_{(r=2 \sqrt{3} R, \theta=\pi / 6)}=-\frac{5 \Gamma}{24 \sqrt{3} \beta}, \\
& \epsilon_{m+}^{(2)}=\left.U\right|_{(r=2 \sqrt{3} R, \theta=\pi / 2)}=\frac{\Gamma}{6 \sqrt{3} \beta} .
\end{aligned}
$$

The magnetic energy of a chain or ribbon is then $U_{d, s}=\sum_{i=1}^{i_{\max }}\left(n_{b-}^{(i)}(d, s) \epsilon_{m-}^{(i)}+n_{b+}^{(i)}(d, s) \epsilon_{m+}^{(i)}\right)$, in this case the summation index $i$ is the order of the interaction that runs up to the highest order $i_{\max }$. It is remarked that the asymmetric cases are not taken into account in this section. These are any case with a nonsymmetric form, where the lengths of the columns in a ribbon are uneven; some asymmetric cases are presented in Fig. 4. Experimental observations state that the superparamagnetic particles in ribbons aggregate in hexagonal lattices to achieve maximum compaction $[12,13]$. Nevertheless, defects in the lattices may occur, and then the aggregates are not regular in general. Strongly asymmetric ribbons have been observed in experiments when a chain joins a ribbon in the dynamics of the thermodynamic equilibrium regime; these cases are also neglected in this work. By counting $n_{b}^{(1)}$ (short lines) without repetition (see Fig. 3) $n_{b}^{(1)}=3(n-$ $1)-p$ is obtained, where $p=2(s+d-2)$ is the number of particles that defines the perimeter of the aggregate [filled (blue) particles]. For $d=2$ the amount of central and border grains are equal $(n=p)$. For $d=1, n_{b}^{(1)}=s-1$ is retrieved, and $p=2(s-1)$ is meaningless. For instance, the cases $(d, s)=(3,5)$ and $(d, s)=(4,6)$, yield $n_{b}^{(1)}=30$ and $n_{b}^{(1)}=53$, respectively. The amount of first order bonds can be also computed by counting the number of negative bonds [short 
solid (blue) lines] and positive bounds (short dashed lines) for nearest neighbors, $n_{b-}^{(1)}$ and $n_{b+}^{(1)}$, respectively. Then $n_{b}^{(1)}=$ $n_{b-}^{(1)}+n_{b+}^{(1)}$. Similarly for second order interactions [long solid (blue) lines and long dashed lines], $n_{b}^{(2)}=n_{b-}^{(2)}+n_{b+}^{(2)}$. After counting various cases, the formulas are $n_{b-}^{(1)}=d(s-1)$, $n_{b+}^{(1)}=(d-1)(2 s-1), n_{b-}^{(2)}=(d-1)(2 s-3), n_{b+}^{(2)}=(d-$ 2) $s$ for $1<d \leqslant s$ and $n_{b+}^{(2)}=0$ for $d=1$. The entropy of the ribbons is $S_{d, s}=-k_{B} \log \left(A_{d, s} / A\right)$, and then the Gibbs free energy (18) is

$$
F=F_{0}+\sum_{d^{\prime}=1}^{d_{\max }^{\prime}} \sum_{s^{\prime}=d^{\prime}}^{\infty} \frac{N_{d^{\prime}, s^{\prime}}}{d^{\prime} s^{\prime}}\left[U_{d^{\prime}, s^{\prime}}+k_{B} T \log \left(\frac{N_{d^{\prime}, s^{\prime}}}{d^{\prime} s^{\prime} A}\right)\right] .
$$

Note that $U_{d, s}$ do not depend on $N_{d, s}$, so the calculation of $\mu_{d, s}=\left.\left(\partial F / \partial N_{d, s}\right)\right|_{\left(N_{d, s} \neq N_{d^{\prime}, s^{\prime}}, T, V\right)}$ is similar to the previous one in Sec. II. Using delta Kronecker conventions $\mu_{d, s}$ yields

$$
\mu_{d, s}=\frac{1}{d s}\left[k_{B} T \log \left(\frac{\phi_{d, s}}{d s}\right)+U_{d, s}\right]+\mu_{d, s}^{0}
$$

with $\mu_{d, s}^{0}=\mu_{0}+\alpha k_{B} T /(d s)$. In this case $\alpha=$ $\log \left(V / A V_{\odot}\right)>0 \quad$ since $\quad V / A V_{\odot}=\bar{n} / \phi_{0}>1$. In thermodynamic equilibrium and considering $\mu_{1,1}^{0}=\mu_{d, s}^{0}$ yields $\phi_{d, s}=d s \phi_{1,1}^{d s} e^{\beta \epsilon_{m} u(d, s)}$, where $u(d, s)=-U_{d, s} / \epsilon_{m}$ is the normalized dimensionless magnetic energy magnitude. In this section the second order potential energy is employed, which is rearranged as $u^{(2)}(d, s)=d s-1-(d-1)-(d-$ 1) $(2 s-1) / 8+5(d-1)(2 s-3) / 24 \sqrt{3}-(d-2) s / 6 \sqrt{3}$. In the latter, the last term is not taken into account for $d=1$, since second order interactions appear only in ribbons. Then the volume fraction of ribbons of length $s$ and width $d$ is

$$
\phi_{d, s}=d s x^{d s} \frac{\phi_{1,1}}{x} r_{d, s}^{(2)},
$$

where $r_{d, s}^{(2)}=e^{-\beta \epsilon_{m}(d-1)\left[1+\frac{(2 s-1)}{8}-\frac{5(2 s-3)}{24 \sqrt{3}}+\frac{(d-2) s}{6(d-1) \sqrt{3}}\right]}$ is a term that applies only in ribbon cases $(d>1)$. The conservation of magnetic grains in the system [similar to Eq. (3) including ribbons] as a function of $x_{0}=\phi_{0} e^{\beta \epsilon_{m}}$ implies

$$
x_{0}=\sum_{d=1}^{d_{\max }} \sum_{s=d}^{\infty} d s x^{d s} r_{d, s}^{(2)}, \quad \begin{cases}r_{d, s}^{(2)}=1 & \text { for } d=1 \\ r_{d, s}^{(2)}<1 & \text { for } d>1 .\end{cases}
$$

The right-hand side of (24) is obtained by computing each summation term $d=1, \ldots, d_{\max }$. After an expansion in series at $x=1$ the solution for chains [14] is obtained, including additional terms

$$
x_{0}=c_{0}\left(\beta, \epsilon_{m}\right)+\frac{1}{(1-x)^{2}}-\frac{1}{(1-x)}+O(x-1),
$$

where $c_{0}$ evaluated at experimental parameters is a negligible value, and it decreases when the magnetic field increases. The aggregates mean length $\bar{s}$ is then computed using Eqs. (23) and $A_{d, s}=\phi_{d, s} V /\left(d s V_{\odot}\right)=x^{d s} \phi_{1,1} r_{d, s}^{(2)} V / x V_{\odot}$ into (17), which yields

$$
\bar{s}=\frac{1}{a_{0}} \sum_{d=1}^{d_{\max }} \sum_{s=d}^{\infty} s x^{d s} r_{d, s}^{(2)}=c_{1}\left(\beta, \epsilon_{m}\right)+\frac{1}{1-x}+O(x-1),
$$

where $a_{0}=\sum_{d=1}^{d_{\max }} \sum_{s=d}^{\infty} x^{d s} r_{d, s}^{(2)}$. The summation is also computed for each term $d=1, \ldots, d_{\max }$. Expanding the result in powers of $(x-1)$ the last equality in Eq. (26) is obtained. The parameter $c_{1}$ also decreases with the magnetic field and is negligible for experimental values. Using (25) and (26) in the limit $x \rightarrow 1$ the result $\bar{s}=\sqrt{\phi_{0} e^{\beta \epsilon_{m}}}$ is retrieved. The contributions of the ribbons in the calculation are not relevant in order to provide the latter result. The complete first order solutions of (25) and (26) give

$$
\bar{s}=\frac{1}{2}+\sqrt{\frac{1}{4}+\phi_{0} e^{\beta \epsilon_{m}}},
$$

correcting the solution at low values of the magnetic field [see Fig. 1 and Fig. 6 (gray thick curve)]. Two problems in the latter equation are recalled. The first one is the employed volume fraction $\phi_{0}=C_{2}^{2}>1$. The second is that Eq. (27) is valid only for low magnetic field magnitudes in the chains regime.

\section{Magnetic energy per particle}

The normalized magnetic energy per particle of an aggregate $U_{N}$ (similar to $U_{d, s} / \epsilon_{m} n$ ) was computed in Ref. [12] as a function of $n$, taking into account the position of each particle and all the possible interactions in aggregates with grains ordered from bottom to top and from left to right (see Fig. 4), but several asymmetric cases are still neglected. This approach is more complete than the previous in Sec. III B since it includes interactions at any order and it considers some asymmetric cases, for instance, $n=d s+1, n=d s+2$, and $n=d s+3$ for $d=4$. The formula for $U_{N}$ is not useful here since its dependence on $s$ is not provided, although it can be approximated, and it will be employed in order to recompute Eq. (23). In Ref. [12], the computation of $U_{N}$ up to $n=50$ shows that the most stable structure changes from chains to ribbons when the magnetic field magnitude increases. The formulas (25) and (26) are obtained by using the second order energy $u^{(2)}(d, s)$, which does not provide the complete information regarding the change in the most stable aggregate as function of $n$ (see Fig. 5), this is important in the computation of $\bar{s}$. By considering interactions up to second order, the curves do not cross, this means that third and higher order interactions have to be taken into account in order to compare $U_{N}$ and $u(d, n)$ around $n_{d}=30,113$, 263 for $d=2,3$, and 4, respectively. Since the computation of the magnetic energy per particle has been performed in Refs. [12,13], including all the interactions in symmetric and in some nonsymmetric cases, a review of the calculation is presented here and it will be used in Eqs. (23) and (26). Each particle in an aggregate is labeled with the index $i$ (see Fig. 4), related to its position $\vec{\rho}_{i}=\left(\rho_{i, x}, \rho_{i, y}\right)$ where

$$
\begin{aligned}
& \rho_{i, x}(d)=\cos \left(\frac{\pi}{6}\right)([i-1] \bmod d), \\
& \rho_{i, y}(d)=\operatorname{int}\left(\frac{i-1}{d}\right)+\sin \left(\frac{\pi}{6}\right)([i-1] \bmod d) \bmod 2
\end{aligned}
$$




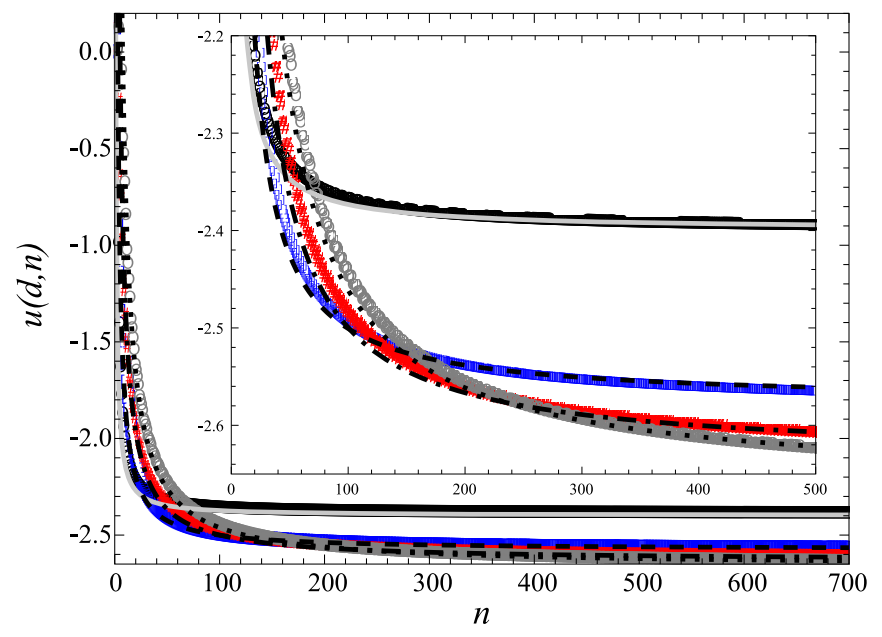

FIG. 5. Normalized magnetic energy per particle $u(d, n)$ of one chain or ribbon computed employing Eq. (29) up to $n=700$ at $d=1$ (circles), $d=2$ [(blue) []], $d=3$ [(red) \#], and $d=4$ (gray circles). Fit $u_{d, n}=2\left(a_{d}-b_{d} n\right) / n$ for $d=1$ (gray line), $d=2$ (dashed curve), $d=3$ (dot-dashed line), and $d=4$ (dotted line). Inset: $u(d, n)$ computed up to $n=500$ particles, the most stable aggregate changes at about $n_{d}=30,113,263$ for $d=2,3,4$ respectively.

are the normalized coordinates of $\vec{\rho}_{i}=\vec{r}_{i} / 2 R$ for $i=1,2 \ldots n$ which is valid for any integer value of $d . a \bmod b$ is the modulus operator (the rest of the integer division $a / b$ ), and int $(x)$ returns the integer part of $x$. The number of particles in an aggregate is related to its length and width by $n=d s+n \bmod d$. The position of the first particle is $(0,0)$, and the grains are ordered from bottom to top and from left to right. The distance between two grains is $\rho_{i j}=\left|\vec{\rho}_{j}-\vec{\rho}_{i}\right|$, and the normalized magnetic energy $u_{0}(i, j)=U(i, j) / U_{0}$ (with $U_{0}=\Gamma / 2 \beta$ ) is obtained employing Eq. (7), then the complete magnetic energy per particle in an aggregate is computed (see Fig. 5) with

$$
u(d, n)=\frac{1}{n} \sum_{i=1}^{n-1} \sum_{j=i+1}^{n} \frac{1}{\rho_{i j}^{3}}\left[1-\frac{3\left(\rho_{j, y}-\rho_{i, y}\right)^{2}}{\rho_{i j}^{2}}\right] .
$$

Previous findings in Ref. [12] suggest that the complete energy of an aggregate $U_{d, s}$ is necessary in order to compute $\bar{s}$. The dependence of the magnetic energy on $s$ can be obtained by approximating the numerical results given by (29). The symmetric approximation $n=d s$ is exact in the cases $n \bmod d=0$ (when $n$ is a multiple of $d$ ), and it is applicable since $0 \leqslant n \bmod d \leqslant d-1 \ll n$. The magnetic energy per grain is computed up to $n=700$ employing Eq. (29). Chains are the most stable aggregate for $n<30$, and ribbons appear at about $n_{2}=30$ for $d=2, n_{3}=113$ for $d=3$, and $n_{4}=263$ for $d=4$. From the latter numerical results a fit for the magnetic energy of an aggregate is obtained by minimizing the difference between $u(d, n)$ and $u_{d, n}=2\left(a_{d}-b_{d} n\right) / n$ for each value of $d$; the coefficients $\left(a_{d}, b_{d}\right)$ are given in Table I. It is taken into account that aggregates with a number of particles $n_{0}=d^{2}$ (dashed line circles, Fig. 4) are the smallest ribbon or chain aligned parallel to the magnetic field direction since $d \leqslant s \ll n$, then the fit is performed for each data set (29) starting from $n=n_{0}$ for $d=1, \ldots, 4$. Numerical results for $n<d^{2}$ do not have physical meaning since they represent aggregates aligned perpendicular to the magnetic
TABLE I. List of coefficients that depend on $d$. The order of the numerical solutions $o_{\max }$ depends on $d_{\max }$.

\begin{tabular}{lcccrr}
\hline \hline$d \backslash d_{\max }$ & $a_{d}$ & $b_{d}$ & $s_{d}$ & $n_{d}$ & $o_{\max }$ \\
\hline 1 & 1.30536 & 1.19968 & 2 & 1 & 2 \\
2 & 3.76001 & 1.28793 & 5 & 30 & 8 \\
3 & 6.69634 & 1.31679 & 6 & 113 & 18 \\
4 & 10.02290 & 1.33089 & 7 & 263 & 32 \\
\hline \hline
\end{tabular}

field direction. The numerical results of (29) at $d=1$ (circles), $d=2$ [(blue) []], $d=3$ [(red) \#] and $d=4$ (gray circles) are fitted in good agreement with $u_{d, n}$ at $d=1$ (gray line), $d=2$ (dashed curve), $d=3$ (dot-dashed curve), and $d=4$ (dotted line). The latter fit is a coarse-grained approach, which is equivalent to the symmetric approximation in the sense that it does not describe the details obtained from the rest $n \bmod d$ particles observed in the numerical results (local groups of data for $d>1$; see inset of Fig. 5). The explicit dependency of $a_{d}$ and $b_{d}$ on $d$ and $s$ can be provided with an approach such as the one given in Sec. IIIB, with a development up to fourth or higher order. The symmetric approximation $n=d s$ is used in the magnetic potential, then $U_{d, s}=n U_{0} u_{d, n}=\epsilon_{m}\left(a_{d}-b_{d} d s\right)$, and the complete volume fraction [similar to Eq. (23)]

$$
\phi_{d, s}=d s x^{d s} \frac{\phi_{1,1}}{x} r_{d, s}
$$

is provided with $r_{d, s}=\exp \left[\beta \epsilon_{m}\left(1-a_{d}+\left(b_{d}-1\right) d s\right)\right]$, and an onset value $s_{d}=\operatorname{int}\left[\left(a_{d}-1\right) /\left(b_{d}-1\right) d\right]$ before $r_{d, s}>1$. In Eqs. (24) and (26) the mean length $\bar{s}$ and $x_{0}$ are defined. Due to the coarse-grained approximation, it is possible to compute the series $s_{0 d}$ and $a_{0 d}$ since $\left(a_{d}, b_{d}\right)$ do not depend on $s$; then

$$
\begin{gathered}
s_{0 d}=\sum_{s=d}^{\infty} s x^{d s} r_{d, s}=a_{0 d}\left(d+\frac{\zeta_{d}^{d}}{1-\zeta_{d}^{d}}\right) \\
a_{0 d}=\sum_{s=d}^{\infty} x^{d s} r_{d, s}=e^{\beta \epsilon_{m}\left(1-a_{d}\right)} \frac{\zeta_{d}^{d^{2}}}{1-\zeta_{d}^{d}}
\end{gathered}
$$

with $\bar{s}=s_{0} / a_{0}, a_{0}=\sum_{d=1}^{d_{\max }} a_{0 d}, s_{0}=\sum_{d=1}^{d_{\max }} s_{0 d}, x_{0}=\sum_{d=1}^{d_{\max }} x_{0 d}$, $x_{0 d}=d s_{0 d}$, and $\zeta_{d}=x e^{\beta \epsilon_{m}\left(b_{d}-1\right)}$. By truncation of the series $a_{0}, s_{0}$ and $x_{0}$ in the chain case $d_{\max }=1$, and using $a_{1}=b_{1}=1$ it is obtained $r_{1, s}=1, \zeta=x, \bar{s}=(1-x)^{-1}, x_{0}=x(1-x)^{-2}$, and the solution (27) is retrieved. In Table I we see that $1<$ $b_{d}<a_{d}$, which provides the complete solution in the case $d_{\max }=1$, it is obtained $\bar{s}(\bar{s}-1)=x_{a 1}$ employing Eqs. (31) and (32), giving

$$
\bar{s}_{a 1}=\frac{1}{2}+\sqrt{\frac{1}{4}+x_{a 1}}
$$

and $x_{a 1}=\phi_{0} e^{\beta \epsilon_{m} a_{1}}$. The latter result allows us to decrease the value of the volume fraction in absence of magnetic field to $\phi_{0}=0.81$ [dot-dashed line in Fig. 1 and dot-dashed (red) line in Fig. 6]. In mixed cases (chains and ribbons) $d_{\max }=2,3,4$, a polynomial equation for $\bar{s}$ is obtained by using the series $\bar{s}$ and $x_{0}$. Each $\zeta_{d}$ can be written as $\zeta_{d}=e^{\beta \epsilon_{m} \Delta b_{d}} \zeta_{1}$ where $\Delta b_{d}=b_{d}-b_{1}$. The equations for $\bar{s}$ 


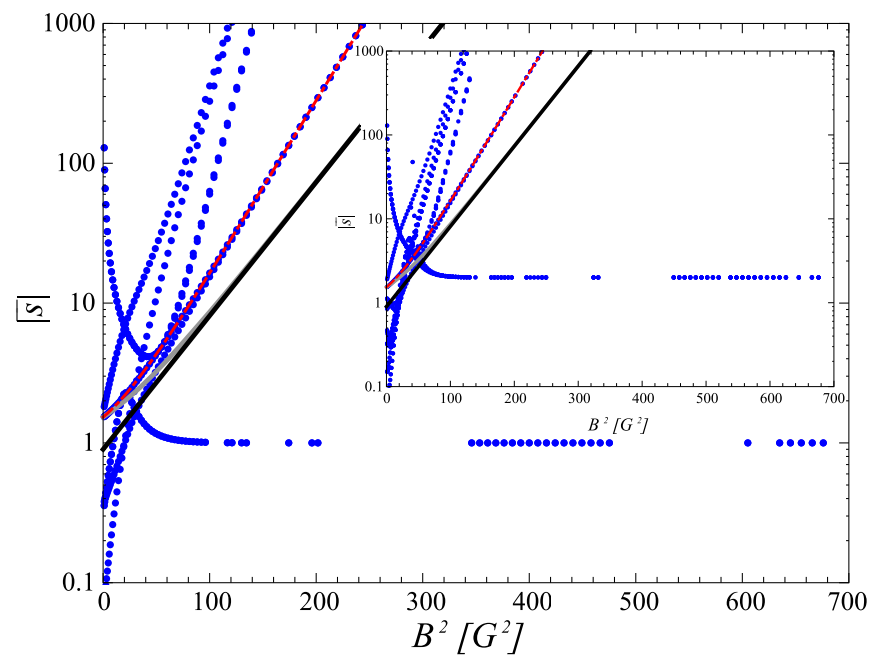

FIG. 6. Mean length of saturated chains and ribbons at thermodynamic equilibrium. Solutions $\bar{s}=\sqrt{\phi_{0} e^{\beta \epsilon_{m}}}$ (black thick line) [12,14], (27) (gray thick curve), and $\bar{s}_{a 1}$ (33) [dot-dashed (red) line] evaluated at $\phi_{0}=0.81$. Absolute value of numerical solutions obtained using (31) and (32) up to $d_{\max }=2$ [(blue) points]. Inset: Absolute value of numerical solutions up to $d_{\max }=3$ ((blue) points).

and $x_{0}$ are written employing Eqs. (31) and (32), which is a set of two polynomials solved numerically for variables $\left(\zeta_{1}, \bar{s}\right)$. The maximum number of solutions (the order of the polynomials) $o_{\max }=8,18,32$ for $\bar{s}$, depends on $d_{\max }=2,3,4$ respectively. The number of solutions also depends on the value of the magnetic field, since two or more solutions can merge at a certain value of $B$, and bifurcate again for a higher value; then the amount of solutions vary in $\left[0, o_{\max }\right]$ for each value of $B$ and $d_{\max }$. The latter explains the numerical intervals with fewer points. The Eqs. (31) and (32) are solved using the original parameter $C_{1}=4.4 \times 10^{-2}\left[G^{-2}\right]$ [12] and the reduced value $C_{2}=\phi_{0}^{1 / 2}=0.9$ for each value of $B$ from $B^{2}=1$ to $B^{2}=700 \mathrm{G}^{2}$ with a step $\Delta B=0.2 \mathrm{G}$. The absolute value of the solutions is computed due to negative and imaginary parts that have no physical sense $(\bar{s} \geqslant 1)$. In Fig. 6 are presented the absolute value of numerical solutions at $d_{\max }=2,3$ obtained using Eqs. (31) and (32) [(blue) points]. Some solutions at $d_{\max }=2$ and at $d_{\max }=3$ match the solution (33) at $d_{\max }=1$ [dot-dashed (red) line], since this case is included in the series $a_{0}, s_{0}$, and $x_{0}$ for $d_{\max } \geqslant 1$. A part of the solutions provide the lower limit $\bar{s}=d_{\max }-1$. It is noticed that the latter bound is a numerical solution without physical sense since $d \leqslant s$. Nevertheless, in a recent work [23] it has been shown that if the mean length $\bar{s}(t)$ is scaled with a characteristic time [21], the time evolution curves $\bar{s}(t)$ collapse and then $\bar{s}$ as a function of the magnetic field is roughly constant, which could be related to the constant numerical solution (see Figs. 6 and 7); this will be the subject of a future work. At $d_{\max }=4$ the number of solutions increases up to the maximum $o_{\max }=32$, we employ $\Delta B=1 \mathrm{G}, \phi_{0}=0.09$, and $C_{1}=4.4 \times 10^{-2} \mathrm{G}^{-2}$. It is observed that the magnitude of the solutions decreases by reducing the monomers volume fraction from $\phi_{0}=0.81$ [(green) \#] to $\phi_{0}=0.09$ (circles) (see Fig. 7). It is possible to reduce the magnitude of $\phi_{0}$ to the experimental value $\phi_{0}=2 \times 10^{-3}$ by using the

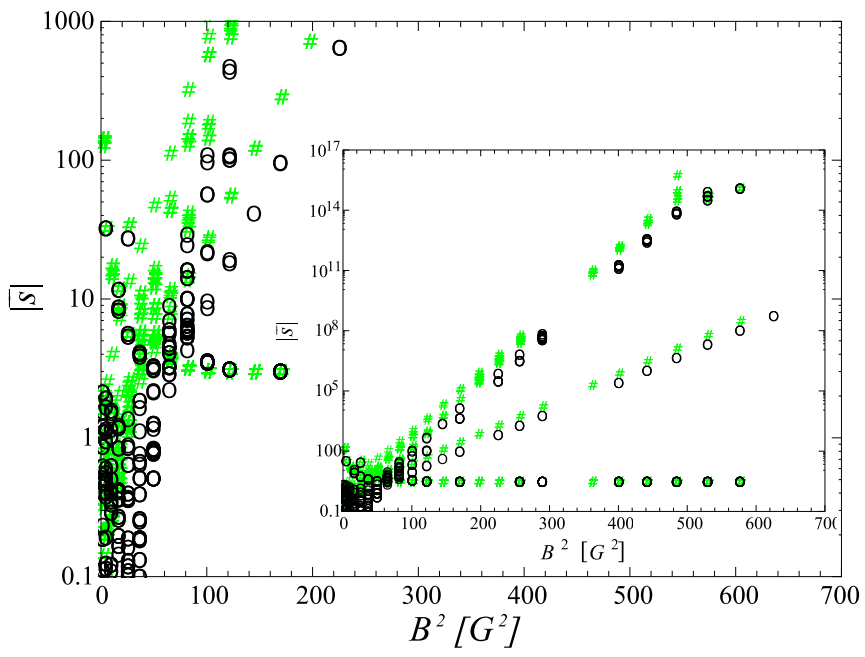

FIG. 7. Mean length of saturated chains and ribbons at thermodynamic equilibrium. Absolute value of numerical solutions obtained using (31) and (32) up to $d_{\max }=4$ for $\phi_{0}=0.81$ [(green) \#]. Absolute value of numerical solutions for $\phi_{0}=0.09$ (circles). Inset: Numerical solutions diverge as $B$ increases. The magnitude of $|\bar{s}|$ grows with $\phi_{0}[24]$.

average magnetic energy $\epsilon_{m}=(\Gamma-1) / \beta$; nevertheless the numerical solutions do not match experimental results in the multistable region by including the complete magnetic interaction energy. The latter will be achieved by considering $K_{d, s} \neq 1$, presented in Sec. III E. Since some solutions match $\bar{s}_{a 1}$ (33), an approximation similar to Eq. (13) is performed using (31), (32), (33), and $\zeta_{1} \simeq 1-\bar{s}_{a 1}^{-1}$ into

$$
\begin{gathered}
\bar{s}=\frac{s_{01}}{a_{01}}=\frac{1}{1-\zeta_{1}} \simeq \sum_{i=0}^{\bar{s}_{\infty}-1} \zeta_{1}{ }^{i} \simeq \sum_{i=0}^{\bar{s}_{\infty}-1}\left(1-\frac{1}{\bar{s}_{a 1}}\right)^{i} \\
\geqslant \sum_{i=0}^{\bar{s}_{\infty}-1}\left(1-\frac{1}{\sqrt{e^{-\beta \epsilon_{m}\left(b_{1}-1\right)} x_{a 1}}}\right)^{i} \gtrsim 1 .
\end{gathered}
$$

Equations (33) (dot-dashed line) and (34) (dashed black curve) depend only on $a_{1}$ (see Fig. 1). Equation (35) is obtained with $x_{0}=s_{01}$ and (31) in the limit $x \rightarrow 1$ (black thin lines); in the case $B=0$ it yields $\bar{s} \simeq 1$, and it depends on $a_{1}$ and $b_{1}$, which is a lower bound of (34) for $b_{1}=1.19968$.

\section{Discussion}

The latter results obtained by including the complete magnetic interaction energy contain part of the physics involved in the structure of the aggregates but lack three important aspects, (a) Although one point at $\bar{s}_{\infty}$ allows us to draw a curve for $\bar{s}$, this does not explain the multistable region or the range of asymptotic values for $\bar{s}_{\infty}$, which depend on the experimental parameters, (b) the values of $\phi_{0}$ employed in analytic and numerical results are much greater than the experimental value, and (c) the numerical findings including the magnetic interaction energy up to $d_{\max }=4$ do not match the experimental data in the multistable region. An ideal result is to obtain a set of two polynomials [such as Eqs. (31) and (32)] solved numerically for the variables $\left(\zeta_{1}, \bar{s}\right)$ with asymptotic solutions at $B \gg 1 G$. The divergence 
of the previous numerical results can be attributed to a number of approximations made in Secs. III B and III C, these are, for instance, the following: (1) Considering aggregates with a number of particles $n=d s+n \bmod d$ and neglecting cases such as completely nonsymmetric ribbons built when a chain leaves a ribbon and merges with another one, as a typical behavior of dynamical equilibrium [12]. (2) Considering the mean interaction free energy per particle as a constant $\mu_{1,1}^{0}=\mu_{d, s}^{0}$. (3) The formation process of the aggregates has not been addressed. This can be studied numerically (Langevin dynamics simulations $[14,19,20]$ ) and theoretically (Smoluchowsky rate equations $[16,17,21]$ ). (4) The respective cases of the formation process and energy barriers should be taken into account [25]. (5) Irreversible aggregation due to electrostatic stabilization could be important, although the reversibility of the aggregation has been shown in experiments [13] by measuring the density of particles in a chain after the magnetic field is turned off.

\section{E. The equilibrium constant}

In this section the previous points (a), (b), (c), and (2) in Sec. III D will be addressed. In a similar procedure as in (22), the thermodynamic equilibrium equation $\mu_{d, s}=\mu_{1,1}$ holds. Now $\mu_{d, s}^{0}$, the standard part of the chemical potential (the mean interaction free energy per particle) is not neglected, and employing the symmetric approximation $n=d s$ the volume fraction $\phi_{d, s}$ yields

$$
\phi_{d, s}=d s x^{d s} \frac{\phi_{1,1}}{x} r_{d, s} K_{d, s},
$$

where $x=\phi_{1,1} e^{\Gamma}$ and $K_{d, s}=e^{-\beta d s\left(\mu_{d, s}^{0}-\mu_{1,1}^{0}\right)}$ is known as the equilibrium constant according to the generalized mass action law. Since $\mu_{d, s}^{0}=\mu_{0}+\alpha /(\beta d s)$ and $\alpha=\log \left(V / A V_{\odot}\right)=$ $\log \left(\bar{n} / \phi_{0}\right)>0$, then $K_{d, s}=\left(\bar{n} / \phi_{0}\right)^{d s-1}$. Several approximations for $\bar{n}$ in $K_{d, s}$ can be employed. In previous sections $K_{d, s}=1$, which is equivalent to consider $\bar{n}=\phi_{0}$. This is a very rough approximation since $\bar{n} \gg \bar{s}>1$ and $0<\phi_{0}<1$. Using Eqs. (16), (17), and (36) the mean number of particles and length of the aggregates are $\bar{n}=x_{0} / a_{0}$ and $\bar{s}=s_{0} / a_{0}$, where $a_{0}, s_{0}$, and $x_{0}$ are defined following Eqs. (31) and (32). In this case $a_{0 d}=\sum_{s=d}^{\infty} x^{d s} r_{d, s} K_{d, s}, s_{0 d}=\sum_{s=d}^{\infty} s x^{d s} r_{d, s} K_{d, s}$ and $x_{0 d}=d s_{0 d}$. Equation (31) remains unchanged and (32) is scaled with the factor $\phi_{0} / \bar{n}$ :

$$
a_{0 d}=\frac{\phi_{0}}{\bar{n}} e^{\Gamma\left(1-a_{d}\right)} \frac{\zeta_{d}^{d^{2}}}{1-\zeta_{d}^{d}},
$$

where $\zeta_{d}=\left(\bar{n} / \phi_{0}\right) x e^{\Gamma\left(b_{d}-1\right)}$ in this case. In this section is considered the approximation $\bar{n} \simeq d \bar{s}$ and a constant value for $\bar{s} \simeq 50$ in $K_{d, s}$, then $\zeta_{d} \simeq d e^{\Gamma \Delta b_{d}} \zeta_{1}$. The numerical step $\Delta B=1 \mathrm{G}$, the experimental value $\phi_{0}=0.002$, and a lower value of $C_{1}=0.2 \times 10^{-2} \mathrm{G}^{-2}$ are employed (see Fig. 8). The height of the chamber is approximately $50 \mu \mathrm{m}$, about 25 to 100 particle diameters. However, the particles are located mostly in a layer of about $2 \mu \mathrm{m}$ near the bottom of the chamber due to sedimentation. The effective volume fraction is then estimated between 0.002 and 0.085 due to thermal fluctuations of the thickness of the sedimentation layer and a wide distribution of particle radii, in agreement with the employed value of $\phi_{0}$. The original value of $C_{1}=4.4 \times 10^{-2} \mathrm{G}^{-2}$ employed

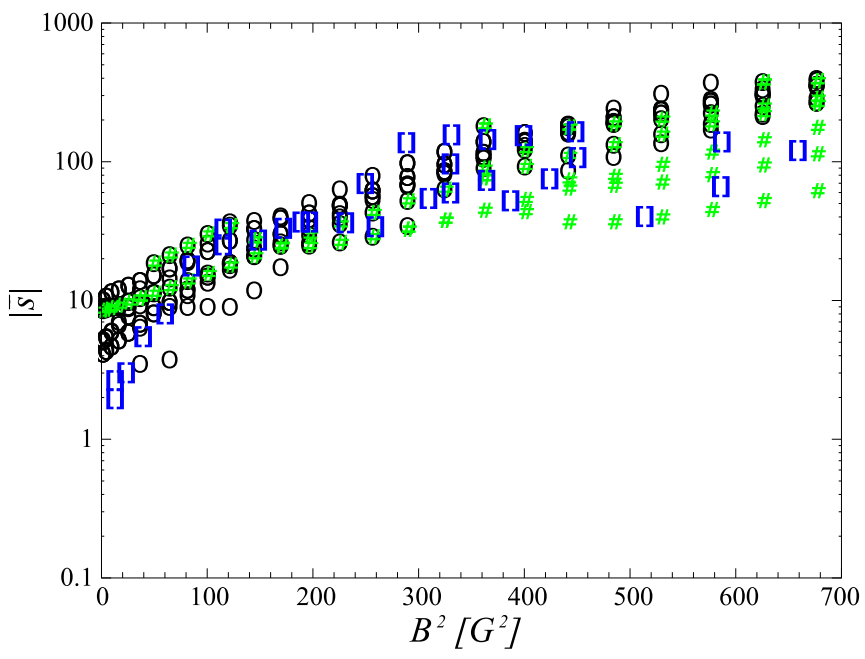

FIG. 8. Mean length of saturated chains and ribbons at thermodynamic equilibrium. Experimental data obtained from Refs. [12,13] [(blue) []]. Absolute value of selected solutions $\bar{s}_{4}$ to $\bar{s}_{12}$ (circles) and completely real numerical data from solutions $\bar{s}_{4}$ to $\bar{s}_{18}$ [(green) \#] using (31) and (37) up to $d_{\max }=4$ for $\phi_{0}=0.002$ and $C_{1}=$ $0.2 \times 10^{-2}\left[G^{-2}\right]$.

previously [12] could only be justified by using the upper and lower values of the measures of $\chi$ and $R$. By using the mean values of these independent measurements, a value $C_{1}=0.12 \times 10^{-2} \mathrm{G}^{-2}$ is obtained. Therefore the employed value of $C_{1}=0.2 \times 10^{-2} \mathrm{G}^{-2}$ corresponds to the experimental mean particle measurements of $\chi$ and $R$. The multiplicity of the solutions rises up to the maximum $o_{\max }=32$. The absolute value of the selected solutions $\bar{s}_{4}$ to $\bar{s}_{12}$ (circles) and the real positive numerical data from solutions $\bar{s}_{4}$ to $\bar{s}_{18}$ [(green) \#] are compared with experimental measurements [(blue) []]. The multistability of the experimental findings is provided by the multiplicity of pure real positive numerical solutions and the absolute value of complex solutions, which increases with the magnetic field magnitude. The lack of precision of the numerical data for low values of $B$ is improved by computing the absolute value of the selected solutions, which provides more accurate results in the range $0<B \lesssim 10 \mathrm{G}$.

\section{SUMMARY AND CONCLUSIONS}

In Sec. III A a mathematical approach is presented considering that the total number of particles in the system is conserved, which is used in following sections. In Sec. III B the magnetic interaction potential up to second order is introduced. The first analytic results considering symmetric aggregates are obtained. Section III C shows the first numerical and analytic results employing the complete magnetic interaction potential and a coarse grained approximation for $d_{\max }=1,2,3$ and $d_{\max }=4$, considering in addition some asymmetric aggregates. In Sec. III D the assets and deficiencies of the obtained findings are reviewed. Points (1), (3), and (4) will be studied in future research. In Sec. III E the mean interaction free energy per particle $\mu_{d, s}^{0}$ is taken into account [point (2) Sec. III D] by employing the approximation $K_{d, s}=\left(50 d / \phi_{0}\right)^{d s-1}$ in good agreement with experimental measurements (see Fig. 8), here the multistability of experimental data for superparamagnetic 
ribbons in thermodynamic equilibrium is described by the multiplicity of the numerical results, which increase with the magnetic field magnitude. The equation $\bar{n}=x_{0} / a_{0}$ can be added to the set $\bar{s}=s_{0} / a_{0}, x_{0}=\sum_{d=1}^{4} x_{0 d}$. These equations will be solved numerically for $\left(\zeta_{1}, \bar{s}, \bar{n}\right)$ in future work. In the latter case the multiplicity of the solutions decreases to the maximum $o_{\max }=22$.

\section{ACKNOWLEDGMENTS}

N.R. acknowledges financial support of the Université de Liège. A.D. is financially supported by Fonds De La Recherche Scientifique - FNRS as a research fellow. This work was financially supported by the Fonds De La Recherche Scientifique - FNRS (Grant PDR T.0043.14) and Université de Liège (Starting Grant C-13/88).
[1] P. C. Jordan, Mol. Phys. 25, 961 (1973).

[2] P. C. Jordan, Mol. Phys. 38, 769 (1979).

[3] M. A. Osipov, P. I. C. Teixeira, and M. M. T. da Gama, Phys. Rev. E 54, 2597 (1996).

[4] J. M. Tavares, J. J. Weis, and M. M. T. da Gama, Phys. Rev. E 59, 4388 (1999).

[5] P. I. C. Teixeira, J. M. Tavares, and M. M. T. da Gama, J. Phys.: Condens. Matter 12, R411 (2000).

[6] A. Y. Zubarev and L. Y. Iskakova, Phys. Rev. E 61, 5415 (2000).

[7] K. I. Morozov and M. I. Shliomis, in Magnetically Controllable Fluids and their Applications, edited by S. Odenbach (SpringerVerlag, New York, 2003), pp. 162-184.

[8] A. Y. Zubarev and L. Y. Iskakova, Phys. Rev. E 68, 061203 (2003).

[9] V. S. Mendelev and A. O. Ivanov, Phys. Rev. E 70, 051502 (2004).

[10] A. Zubarev, J. Fleischer, and S. Odenbach, Physica A 358, 475 (2005).

[11] L. Y. Iskakova, G. A. Smelchakova, and A. Y. Zubarev, Phys. Rev. E 79, 011401 (2009).

[12] A. Darras, J. Fiscina, M. Pakpour, N. Vandewalle, and G. Lumay, Eur. Phys. J. E. 39, 47 (2016).

[13] A. Darras, Auto-organisation sous champ constant de colloïdes superparamagnetiques, M.Sc. thesis, Université de Liège (2016).

[14] J. S. Andreu, J. Camacho, and J. Faraudo, Soft Matter 7, 2336 (2011).
[15] J. Faraudo, J. S. Andreu, and J. Camacho, Soft Matter 9, 6654 (2013).

[16] F. Martínez-Pedrero, M. Tirado-Miranda, A. Schmitt, and J. Callejas-Fernández, Phys. Rev. E 76, 011405 (2007).

[17] F. Martínez-Pedrero, A. El-Harrak, J. C. Fernández-Toledano, M. Tirado-Miranda, J. Baudry, A. Schmitt, J. Bibette, and J. Callejas-Fernández, Phys. Rev. E 78, 011403 (2008).

[18] G. Odriozola, A. Schmitt, J. Callejas-Fernández, R. MartínezGarcía, and R. Hidalgo-Álvarez, J. Chem. Phys. 111, 7657 (1999).

[19] P. Domínguez-García, S. Melle, J. M. Pastor, and M. A. Rubio, Phys. Rev. E 76, 051403 (2007).

[20] V. Schaller, U. Kräling, C. Rusu, K. Petersson, J. Wipenmyr, A. Krozer, G. Wahnström, A. Sanz-Velasco, P. Enoksson, and C. Johansson, J. Appl. Phys. 104, 093918 (2008).

[21] J. H. E. Promislow, A. P. Gast, and M. J. Fermigier, J. Chem. Phys. 102, 5492 (1998).

[22] J. N. Israelachvili, Intermolecular and Surface Forces, 2nd ed. (Academic Press, New York, 1992), pp. 341-350.

[23] A. Darras, M. Pakpour, N. Vandewalle, G. Lumay, and J. Fiscina (unpublished).

[24] The following values of $\left(a_{d}, b_{d}\right)$ were used in order to improve the performance of the numerical results in this case: $a_{1}=1.55, a_{2}=4.1, a_{3}=7.1, a_{4}=10.4, b_{1}=1.19968$ $b_{2}=1.28968, b_{3}=1.31668, b_{4}=1.32968$.

[25] R. Messina and L. Spiteri, Eur. Phys. J. E 39, 81 (2016). 